\title{
Penetration of Experimental Infiltrants with Different Penetration Coefficients and Ethanol Addition into Natural Caries Lesions in Primary Molars
}

\author{
S. Paris ${ }^{a}$ V.M. Soviero ${ }^{d}$ A.J. Chatzidakis ${ }^{b}$ H. Meyer-Lueckel ${ }^{c}$ \\ ${ }^{a}$ Clinic for Conservative Dentistry and Periodontology, School of Dental Medicine, Christian-Albrechts-Universität \\ zu Kiel, Kiel, and ' ${ }^{b}$ Department of Prosthetic Dentistry, School of Dental Medicine, Charité Centrum 3, Charité - \\ Universitätsmedizin Berlin, Berlin, and ${ }^{\mathrm{C}}$ Department of Operative Dentistry, Periodontology and Preventive Dentistry, \\ Rheinisch-Westfälische Technische Hochschule (RWTH) Aachen, Aachen, Germany; ${ }^{\mathrm{d}}$ Department of Preventive and \\ Community Dentistry, Faculty of Dentistry, Universidade do Estado do Rio de Janeiro, Rio de Janeiro, Brazil
}

\section{Key Words}

Caries - Infiltration $\cdot$ Micro-invasive treatment $\cdot$ Penetration coefficient

\begin{abstract}
This study evaluated the influence of the penetration coefficient (PC) and ethanol addition on the penetration depth (PD) of experimental infiltrants into proximal caries lesions in primary molars. Caries lesions $(n=45)$ were randomly treated with 1 of 4 experimental infiltrants (PC63; PC185; PC204; PC391) for $5 \mathrm{~min}$. Lesion depths and PDs were analysed using dual fluorescence confocal microscopy. Lesions were almost completely infiltrated in all groups. Median PDs and percentage penetrations were not significantly different between groups ( $p>0.05$ ). When applied for $5 \mathrm{~min}$, all tested infiltrants were able to infiltrate proximal caries in primary molars nearly completely.

Copyright $\odot 2012$ S. Karger AG, Basel
\end{abstract}

Non-cavitated lesions in the occlusal surface can be effectively controlled with fissure sealants [Llodra et al., 1993; Simonsen, 2002; Griffin et al., 2008]. Promising results have also been observed after sealing enamel lesions in smooth surfaces but the technique requires temporary tooth separation for several days [Martignon et al., 2006]. An alternative is to infiltrate the lesion body with low-viscosity resins (infiltrants). While sealing creates a protective resin layer on the tooth surface, the caries infiltration technique aims to occlude the pores within the lesion body with resin, impeding the continuous diffusion of acids and dissolved minerals through the lesion, and thus hampering caries progression [Meyer-Lueckel and Paris, 2008b]. Initial lesions in smooth surfaces infiltrated with low-viscosity resins became more resistant to further demineralization, and the ability to inhibit lesion progression was strongly correlated with the penetration depth (PD) of the resin [Meyer-Lueckel and Paris, 2008b]. As adhesives and sealants only shallowly infiltrated natural caries lesions, even after etching the lesion surface with $\mathrm{HCl}$ [MeyerLueckel and Paris, 2008a], low-viscosity resins were tested aiming to enhance infiltration [Paris et al., 2007a]. Caries infiltration has been shown to inhibit caries progression under clinical conditions both in primary [Ekstrand et al., 2010] and permanent teeth [Paris et al., 2010b].

The penetration of low-viscosity resins into the pores of the lesion body is mainly driven by capillary forces and depends on the penetration time, the capillary radius and the penetration coefficient (PC) of the liquid. The PC combines the liquid properties viscosity, surface tension and contact angle to the solid [Fan et al., 1975]. It has been

\section{KARGER}

Fax +4161306 1234

E-Mail karger@karger.ch

www.karger.com
(C) 2012 S. Karger AG, Basel

$0008-6568 / 12 / 0462-0113 \$ 38.00 / 0$

Accessible online at:

www.karger.com/cre
Dr. Vera Mendes Soviero

Clinic for Conservative Dentistry and Periodontology, School of Dental Medicine Christian-Albrechts-Universität zu Kiel

Arnold-Heller-Strasse 3, Haus 26, DE-24105 Kiel (Germany)

Tel. +49431597 2817, E-Mail verasoviero@gmail.com 
Table 1. Composition of experimental infiltrants

\begin{tabular}{lllllll}
\hline Material & \multicolumn{2}{l}{ Ingredients, \% } & \multicolumn{2}{c}{ PC, cm/s } \\
\cline { 2 - 5 } & BIS-GMA & TEGDMA & ethanol & camphorquinone & DABE & \\
\hline PC63 & 24.75 & 74.25 & - & 0.5 & 0.5 & 63 \\
PC185 & 19.8 & 59.4 & 19.8 & 0.5 & 0.5 & 185 \\
PC204 & - & 99.0 & - & 0.5 & 0.5 & 204 \\
PC391 & - & 79.2 & 19.8 & 0.5 & 0.5 & 391 \\
\hline
\end{tabular}

BIS-GMA = Bisphenol-A-glycerol dimethacrylate; TEGDMA = triethyleneglycol dimethacrylate; DABE = ethyl-4-(dimethylamino)benzoate. PCs were the same as used in a previous study with permanent teeth [Meyer-Lueckel and Paris, 2010].

observed that the PD achieved in a specific application time is highly dependent on the PC of the resin [Paris et al., 2007a].

Infiltrants with different PCs and different concentrations of ethanol were tested in natural caries lesions in permanent teeth and deepest and most homogeneous infiltration was obtained with a solvent-free resin with high PC $(204 \mathrm{~cm} / \mathrm{s})$ [Meyer-Lueckel and Paris, 2010]. As the deciduous enamel is relatively different from the permanent enamel concerning thickness, ultrastructure and mineral composition [Mortimer, 1970; Lindén et al., 1986], rather different results might be expected with primary teeth. Therefore, the aim of the present study was to evaluate if the $\mathrm{PD}$ of an infiltrant into natural proximal caries in primary molars is influenced by its PC or by the presence of ethanol as solvent.

\section{Materials and Methods}

Sixty extracted or exfoliated primary molars showing active (chalky opacity, dull surface) non-cavitated proximal lesions scored as ICDAS 2 [Ismail et al., 2007] were selected for the study. Teeth were stored in $0.1 \%$ thymol solution up to usage.

Lesions were etched with 15\% HCl gel (Icon pre-product; DMG, Hamburg, Germany) for 2 min and subsequently rinsed with water spray for $30 \mathrm{~s}$. After drying, lesions were stained with $0.1 \%$ ethanolic solution of tetramethylrhodamine isothiocyanate (Sigma-Aldrich, Steinheim, Germany) for $12 \mathrm{~h}$ and dried with compressed air for $10 \mathrm{~s}$ [Paris et al., 2009]. Teeth were randomly allocated to 4 treatment groups according to the experimental infiltrants (PC63, PC185, PC204 or PC391; table 1). The infiltrants were applied on the lesion for $5 \mathrm{~min}$. After removing excess material from the tooth surface with a cotton roll, the resin was light cured for $60 \mathrm{~s}$ (400 mW/cm² ${ }^{2}$ Translux CL; Heraeus Kulzer, Hanau, Germany).

Tooth sections with $1 \mathrm{~mm}$ thickness were prepared perpendicularly to the lesion surface (Exakt 300cl; Exakt Apparatebau, Norderstedt, Germany), fixed on microscope slides and polished plano parallel (Mikroschleifsystem 400 cs, Abrasive Paper 1200, 2400, 4000; Exakt Apparatebau). Unbound red fluorophore dye was bleached by immersion in hydrogen peroxide (30\%) for $24 \mathrm{~h}$ at $37^{\circ} \mathrm{C}$. Subsequently specimens were washed with water. To label porous structures which were not infiltrated, specimens were immersed in a $50 \%$ ethanol solution of $100 \mu \mathrm{M}$ sodium fluorescein (Sigma-Aldrich) for $3 \mathrm{~min}$ and subsequently washed with deionized water for $10 \mathrm{~s}$.

Specimens were observed with a confocal laser scanning microscope (Leica, TCS NT; Leica, Heidelberg, Germany) using a $\times 10$ objective in dual fluorescence mode as described previously [Paris et al., 2009]. In confocal laser scanning microscopy (CLSM) images, infiltrated structures appeared red (methylrhodamine isothiocyanate), and porous structures (not infiltrated enamel or dentin) appeared green (sodium fluorescein). Lesion depth (LD) in enamel was measured from the surface to the deepest point of green fluorescence in enamel. The PD of the infiltrant was measured from the surface to the deepest point of red fluorescence at the point where LD was measured. Measurements were made using Image (NIH, Bethesda, Md., USA). The evaluator was blind with regard to group allocation of the teeth. CLSM was validated previously as a method to evaluate caries infiltration with excellent inter- and intra-examiner reproducibility in natural caries lesions [Paris et al., 2009].

SPSS (SPSS Inc., Chicago, Ill., USA) was used to perform statistical analysis. $\mathrm{PD}$ and percentage penetration $(\mathrm{PP}=\mathrm{PD} / \mathrm{LD} \times$ 100) were compared between the different experimental infiltrants. Subgroup analysis was done for lesions with maximum LD higher than $400 \mu \mathrm{m}$. Assumption of normal distribution was checked using the Shapiro-Wilk test. Differences in LD, PD and PP were analysed using Kruskal-Wallis and Mann-Whitney tests. The level of significance was set at $5 \%$.

\section{Results}

Fifteen teeth were excluded either because they broke during preparation or they were too shallow (less than $150 \mu \mathrm{m})$. The final sample consisted of 45 teeth. 
Table 2. Maximum LD and PD ( $\mu \mathrm{m})$ of the 4 infiltrant PCs for all lesions and for lesions with LD $\geq 400 \mu \mathrm{m}$

\begin{tabular}{|c|c|c|c|c|c|c|}
\hline \multirow[t]{2}{*}{ Material } & \multicolumn{3}{|c|}{ All lesions } & \multicolumn{3}{|c|}{ Lesion with $\mathrm{LD} \geq 400 \mu \mathrm{m}$} \\
\hline & $\mathrm{n}$ & LD & $\mathrm{PD}$ & $\mathrm{n}$ & LD & PD 0-399 $\mu \mathrm{m}$ \\
\hline PC63 & 11 & $457(314 ; 481)$ & $359(202 ; 463)$ & 7 & $465(457 ; 664)$ & $420(202 ; 481)$ \\
\hline PC185 & 12 & $437(390 ; 550)$ & $383(199 ; 450)$ & 9 & $467(409 ; 587)$ & $413(221 ; 519)$ \\
\hline PC204 & 12 & $452(264 ; 490)$ & $407(264 ; 490)$ & 7 & $477(457 ; 514)$ & $477(436 ; 514)$ \\
\hline РC391 & 10 & $462(272 ; 552)$ & $428(254 ; 538)$ & 7 & $504(455 ; 639)$ & $469(401 ; 639)$ \\
\hline $\mathrm{p}$ & & $>0.05$ & $>0.05$ & & $>0.05$ & $>0.05$ \\
\hline
\end{tabular}

The Mann-Whitney test was used to compare medians between groups; $\mathrm{n}=$ sample size per group; 25 th and 75th percentiles are given in parentheses.

The majority of the proximal lesions were deeply infiltrated irrespectively of the infiltrant PC or ethanol addition. The median LD was $457 \mu \mathrm{m}$ (25th percentile 310; 75 th percentile 499$)$ for all lesions $(n=45)$. No significant differences were observed between groups regarding LD ( $p>0.05$; Kruskal-Wallis test; table 2). Median PD and PP did not differ significantly between the 4 experimental infiltrants ( $p>0.05$; Kruskal-Wallis test; table 2).

In the subgroup analysis including only lesions $\geq 400$ $\mu \mathrm{m}(\mathrm{n}=30)$, the median LD was $474 \mu \mathrm{m}$ (25th percentile 457 ; 75 th percentile 582 ). No significant differences concerning LD were observed between the groups ( $\mathrm{p}>0.05$; Kruskal-Wallis test; table 2). Median PD (table 2) and PP (fig. 1) were not significantly influenced by PC or ethanol addition ( $\mathrm{p}>0.05$; Kruskal-Wallis test), although some lesions were partially penetrated with PC63 (without ethanol) and with PC185 (with ethanol).

\section{Discussion}

In the current in vitro study, PD of an infiltrant into natural caries lesions in primary molars was not significantly influenced by the infiltrant PC. Proximal caries lesions were deeply infiltrated even when an infiltrant with low PC $(63 \mathrm{~cm} / \mathrm{s})$ was used. In a previous study with permanent teeth, infiltrants with higher PCs penetrated significantly deeper into caries lesions than infiltrants with low PCs [Meyer-Lueckel and Paris, 2010].

Although the PD of an infiltrant into enamel caries is highly dependent on the PC of the resin [Paris et al., 2007a], other variables like application time, LD and pore dimensions also influence the PD.

Due to de- and remineralization processes in the oral cavity, the surface layer of natural caries lesions is more

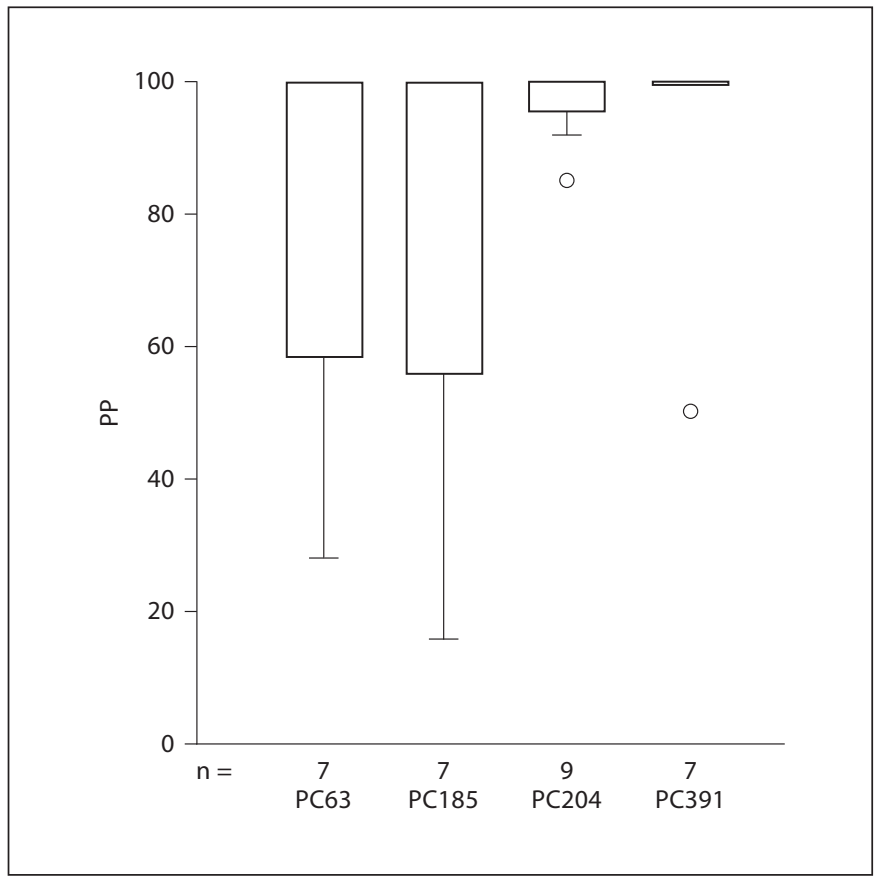

Fig. 1. Box plots of the PP of the 4 experimental infiltrants for lesions with LD $\geq 400 \mu \mathrm{m}$ ( $\mathrm{p}>0.05$; Mann-Whitney test); $\mathrm{n}=$ number of teeth.

highly mineralized and less porous than the subsurface [Holmen et al., 1985]. As caries infiltration aims to occlude the porosities within the lesion body, the surface layer must be perforated or removed otherwise the infiltrant is not able to reach the deepest parts of the caries lesion. Partial removal of the surface layer inhibits resin penetration and results in shallower infiltration [Paris et al., 2007b]. 
Treatment with 15\% hydrochloric acid gel was shown to be the most effective method to prepare the surface of caries lesions for infiltration [Meyer-Lueckel et al., 2007]. While in primary teeth the surface layer was usually completely removed after etching with hydrochloric acid for $2 \mathrm{~min}$ [Paris et al., 2010a], residues of the surface layer remained in permanent teeth [Meyer-Lueckel and Paris, 2008a]. It might be speculated that lesions in primary teeth were more active than in the permanent ones. In more active enamel lesions, the surface layer is expected to be thinner and more susceptible to hydrochloric acid etching. This difference in lesion activity and surface layer thickness could also explain why more complete infiltration was observed in primary teeth even with infiltrants with a low PC compared to permanent teeth, where infiltrants with a lower PC resulted in significantly shallower penetration [Meyer-Lueckel and Paris, 2010]. Another possible explanation for this observation is that lesions in primary teeth were not as deep as lesions in permanent ones and thus were more easily completely infiltrated.

Although no significant differences were observed between the infiltrants with different PCs, some findings are nonetheless worth mentioning. The higher number of lesions partially infiltrated was observed with the infiltrant PC185 (with ethanol). Among lesions deeper than $400 \mu \mathrm{m}$, partial infiltration was also observed with the infiltrant PC63 (without ethanol). Even though infiltrants with lower PCs were also able to penetrate deep into proximal caries in primary molars in the majority of cases, some lesions were only incompletely infiltrated (fig. 1). Only the 2 materials with a higher PC (PC204 without ethanol and PC391 with ethanol) reliably infiltrated the lesions completely (PP $>90 \%)$.

We suppose that infiltrants with low PCs were able to reach the deepest parts of the lesions not only because lesions were probably highly porous, but also because the application time was rather long (5 min). In a recent study with primary teeth, a material with a high PC also showed complete infiltration even after shorter application times [Paris et al., 2011]. As it is preferable to use shorter application times in clinical practice, an infiltrant with a high PC might be preferred not only for permanent, but also for primary teeth.

Volatile solvents, such as ethanol or acetone, are usually added to adhesives to decrease viscosity and improve penetrability [Yiu et al., 2005; Ye et al., 2007; Pashley et al., 2011]. However, deeper penetration into enamel caries in permanent teeth was observed with a solvent-free res- in [Meyer-Lueckel and Paris, 2010]. In the current study, both solvent-free and ethanol-containing resins completely infiltrated the lesions. However, as infiltrants penetrate several hundreds of micrometres into the enamel, solvents might not be completely eliminated by evaporation before light curing, and polymerization may be compromised [Yiu et al., 2005; Ye et al., 2007; Pashley et al., 2011]. Since an infiltrant without ethanol was able to infiltrate caries lesions completely, it is reasonable to recommend that solvent-free resins should be preferable for caries infiltration.

In the current study, just experimental materials were used in order to investigate the influence of PC and solvent on penetrativity. However, the material properties of material PC204 are similar to the commercial product Icon (DMG, Hamburg), which is also solvent free and mainly based on triethylene glycol dimethacrylate, and thus similar results might be expected for this material.

Based on the results of the present in vitro study, it can be concluded that non-cavitated proximal caries lesions in primary molars can be completely infiltrated by lowviscosity resins. When applied for $5 \mathrm{~min}$, a higher PC or ethanol addition did not result in significantly deeper penetration.

\section{Acknowledgments}

The authors are indebted to Mr. Michael Stiebritz for his most valuable contribution to this study and to Prof. Dr. D. Kabelitz, Institute for Immunology, UK-SH, Christian-Albrechts-Universität zu Kiel, for providing the CLSM.

\section{Disclosure Statement}

The Charité - Universitätmedizin Berlin holds US and European patents for an infiltration technique for dental caries lesions in which two of the authors (S.P. and H.M.L.) are appointed as inventors. These patents have been licensed by DMG, Hamburg, Germany. S.P. and H.M.L. receive royalties from this license as well as a research grant from DMG. 


\section{References}

-Ekstrand KR, Bakhshandeh A, Martignon S: Treatment of proximal superficial caries lesions on primary molar teeth with resin infiltration and fluoride varnish versus fluoride varnish only: efficacy after 1 year. Caries Res 2010;44:41-46.

-Fan PL, Seluk LW, O'Brien WJ: Penetrativity of sealants. Part I. J Dent Res 1975;54:262-264.

- Griffin SO, Oong E, Kohn W, Vidakovic B, Gooch BF, Bader J, Clarkson J, Fontana MR, Meyer DM, Rozier RG, Weintraub JA, Zero DT, Group CDSSRW: The effectiveness of sealants in managing caries lesions. J Dent Res 2008;87:169-174.

- Holmen L, Thylstrup A, Ogaard B, Kragh F: A polarized light microscopic study of progressive stages of enamel caries in vivo. Caries Res 1985;19:348-354.

Ismail AI, Sohn W, Tellez M, Amaya A, Sen A, Hasson H, Pitts NB: The international caries detection and assessment system (ICDAS): an integrated system for measuring dental caries. Community Dent Oral Epidemiol 2007;35:170-178.

Lindén LA, Björkman S, Hattab F: The diffusion in vitro of fluoride and chlorhexidine in the enamel of human deciduous and permanent teeth. Arch Oral Biol 1986;31:33-37.

Llodra JC, Bravo M, Delgado-Rodriguez M, Baca P, Galvez R: Factors influencing the effectiveness of sealants - a meta-analysis. Community Dent Oral Epidemiol 1993;21:261268.
Martignon S, Ekstrand KR, Ellwood R: Efficacy of sealing proximal early active lesions: an 18-month clinical study evaluated by conventional and subtraction radiography. Caries Res 2006;40:382-388.

Meyer-Lueckel H, Paris S: Improved resin infiltration of natural caries lesions. J Dent Res 2008a;87:1112-1116.

Meyer-Lueckel H, Paris S: Progression of artificial enamel caries lesions after infiltration with experimental light curing resins. Caries Res 2008b;42:117-124.

Meyer-Lueckel H, Paris S: Infiltration of natural caries lesions with experimental resins differing in penetration coefficients and ethanol addition. Caries Res 2010;44:408-414.

Meyer-Lueckel H, Paris S, Kielbassa AM: Surface layer erosion of natural caries lesions with phosphoric and hydrochloric acid gels in preparation for resin infiltration. Caries Res 2007;41:223-230.

Mortimer KV: The relationship of deciduous enamel structure to dental disease. Caries Res 1970;4:206-223.

- Paris S, Bitter K, Renz H, Hopfenmuller W, Meyer-Lueckel H: Validation of two dual fluorescence techniques for confocal microscopic visualization of resin penetration into enamel caries lesions. Microsc Res Tech 2009;72:489-494.

Paris S, Dörfer CE, Meyer-Lueckel H: Surface conditioning of natural enamel caries lesions in deciduous teeth in preparation for resin infiltration. J Dent 2010a;38:65-71.
Paris S, Hopfenmuller W, Meyer-Lueckel H: Resin infiltration of caries lesions: an efficacy randomized trial. J Dent Res 2010b;89:823826.

-Paris S, Meyer-Lueckel H, Cölfen H, Kielbassa AM: Resin infiltration of artificial enamel caries lesions with experimental light curing resins. Dent Mater J 2007a;26:582-588.

Paris S, Meyer-Lueckel H, Kielbassa AM: Resin infiltration of natural caries lesions. J Dent Res 2007b;86:662-666.

Paris S, Soviero VM, Seddig S, Meyer-Lueckel H: Penetration depths of an infiltrant into proximal caries lesions in primary molars after different application times in vitro. Int $\mathrm{J} \mathrm{Pae}-$ diatr Dent 2011, E-pub ahead of print.

- Pashley DH, Tay FR, Breschi L, Tjäderhane L, Carvalho RM, Carrilho M, Tezvergil-Mutluay A: State of the art etch-and-rinse adhesives. Dent Mater 2011;27:1-16.

Simonsen RJ: Pit and fissure sealant: review of the literature. Pediatr Dent 2002;24:393414.

Ye Q, Spencer P, Wang Y, Misra A: Relationship of solvent to the photopolymerization process, properties, and structure in model dentin adhesives. J Biomed Mater Res A 2007;80: 342-350.

-Yiu CK, Pashley EL, Hiraishi N, King NM, Goracci C, Ferrari M, Carvalho RM, Pashley DH, Tay FR: Solvent and water retention in dental adhesive blends after evaporation. Biomaterials 2005;26:6863-6872. 